\title{
LA INDUSTRIA AZUCARERA Y EL DESARROLLO DOMINICANO
}

\section{INTRODUCCION}

En el pasado año 1980, en la Universidad Central del Este, para los días 21,22 y 23 de noviembre, se celebró un Seminario Nacional sobre "La Industria Azucarera y el Desarrollo Dominicano". El evento fue auspiciado por la Asociadel Este y la Asociación te Tecnóliversidades (ADRU), la Universidad Central (ATAREDO).

En el Seminario participaron numerosas personalidades, técnicos e intelectuales vinculados a la cuestión azucarera, tanto nacionales como extranjeros. Entre otros: Ing. César García, Ing. Felipe Vicini, Sr. José Lagos, Lic. José del Castillo, Lic. Hugo Guilliani Cury, Lic. Altagracia Rivera de Castillo, Dr. Eduardo Latorre, Ing. Eulogio Santaella, Lic. Rafael Toribio, Dr. Wilfredo Lozano. En dicipación a través de tas Tecnológico de Santo Domingo tuvo una destacada partipresentaron.

Seminario auspiciado por la Asociación Dominicana de Rectores Universitarios (ADRU) la Asociación de Tecnólogos Azucareros de la República Dominicana (ATARELO) y la), Universidad Central del Este (UCE) Macorís, R.D. 
Por la importancia que reviste la cuestión azucarera para el desarrollo nacional, como por la elevada calidad-académica de las ponencias y comentarios expuestos, CIENCIA Y SOCIEDAD publica en este número los trabajos presentados por los señores Dr. Eduardo Latorre, Rector del INTEC, Lic. Rafael Toribio, Vicerrector Ejecutivo, Dr. Wilfredo Lozano, Director de Divulgación Científica, Ing. Eulogio Santaella, miembro de la Junta de Regentes; asímismo, se publican las ponencias presentadas por la Lic. Altagracia Rivera de Castillo y por el Lic. José del Castillo, dada la importancia de las problemáticas que abordan y su alta calidad académica. Estas dos úl timas ponencias son las que comentan el Ing. Eulogio Santaella y el Dr. Wilfredo Lozano, respectivamente.

En el evento se presentaron otras ponencias y comentarias de tanta calidad como las aquí publicadas, pero dado nuestro límite de espacio nos hemos visto forzados a realizar una selección de las mismas. CIENCIA Y SOCIEDAD espera con la publicación de dichas ponencias contribuir modestamente al conocimiento de nuestra actual realidad nacional y a la definición de las maneras de superación de nuestro actual estado de subdesarrollo. 